\title{
Extended Epitympanotomy for Facial Nerve Decompression as a Minimally Invasive Approach
}

\author{
Janet Ren Chao ${ }^{1}$, Jiwon Chang ${ }^{2}$, and Jun Ho Lee ${ }^{2}$ \\ ${ }^{1}$ Division of Otolaryngology, Department of Surgery, Yale School of Medicine, New Haven, CT, USA \\ ${ }^{2}$ Department of Otorhinolaryngology and Head and Neck Surgery, College of Medicine, Hallym University, Chuncheon, Korea
}

\author{
Received January 4, 2019 \\ Revised March 5, 2019 \\ Accepted April 1, 2019
}

\author{
Address for correspondence \\ Jun Ho Lee, MD, PhD \\ Department of Otorhinolaryngology \\ and Head and Neck Surgery, \\ Chuncheon Sacred Heart Hospital, \\ College of Medicine, \\ Hallym University, \\ 77 Sakju-ro, Chuncheon 24253, \\ Korea \\ Tel $+82-33-240-5181$ \\ Fax +82-33-241-2909 \\ E-mail_zoonox@nate.com
}

\begin{abstract}
For a minimally invasive approach to access the facial nerve, we designed an extended epitympanotomy via a transmastoid approach that has proven useful in cases of traumatic facial nerve palsy and pre-cholesteatoma. To evaluate the surgical exposure through an extended epitympanotomy, six patients with traumatic facial nerve palsy were enrolled in this study. The same surgical technique was used in all patients. Patients were assessed and the degree of facial nerve paralysis was determined prior to surgery, 1-week post-operatively, and 6-months post-operatively using the House-Brackmann grading system. In all cases, surgical exposure was adequate. All patients with traumatic facial nerve palsy were male and the age range was 13 to 83 years. In all cases, the location of the facial nerve damage was limited to the area between the first and second genu. Symptoms of all the patients improved by 6 months post-operation $(p=0.024)$. There were no complications in any of the patients. Extended epitympanotomy is useful for safe, rapid surgical exposure of the attic area, sparing the patient post-operative dimpling, skin incision complications, and lengthy exposure to anesthesia. We suggest that surgery for patients with facial nerve palsy secondary to trauma be performed using this described technique.

J Audiol Otol 2019;23(4):204-209

KEY WORDS: Middle ear · Mastoid · Middle ear ventilation Minimally invasive surgical procedures.
\end{abstract}

\section{Introduction}

Several approaches can be used to decompress the facial nerve when paralysis has occurred due to trauma. The middle cranial fossa approach, translabyrinthine approach, or transmastoid approach are used depending on the injury site [1-3]. The transcanal approach with an endoscope has been tried as a minimally invasive technique to reach the specific portion of the facial nerve affected by trauma [4]. For mild cases, some authors have recommended conservative treatment with corticosteroids [3].

Among these, the middle cranial fossa approach is used when the traumatic lesion is in the perigeniculate region or in the labyrinthine segment [2]. Hearing preservation is the main advantage of this approach; however, opening the scalp can

This is an Open Access article distributed under the terms of the Creative Commons Attribution Non-Commercial License (https://creativecommons.org/licenses/by-nc/4.0/) which permits unrestricted non-commercial use, distribution, and reproduction in any medium, provided the original work is properly cited. sometimes induce intracranial complications (cerebrospinal fluid leak, seizure, stroke, hematoma) [5]. In cases involving lesions similar to those where the middle cranial fossa approach is used but complete hearing loss in the ipsilateral ear is confirmed, the translabyrinthine approach is used [5]. The transmastoid approach is the gold standard technique when the facial nerve trauma is localized to the tympanic segment or the mastoid segment [3]. Conventionally, the canal wall up mastoidectomy with facial recess opening is the basic technique, and extensive facial nerve exposure by drilling is necessary to decompress the pathologic lesion. However, identification of the facial nerve requires skill and a long operation time, and may result in iatrogenic trauma to the normal portion of the facial nerve.

Mastoidectomy is used to remove pathologic tissue in the mastoid, reduce the ventilating mucosa, or gain access to the epitympanum. It is an essential procedure to treat chronic otitis media (COM) that extends into the mastoid. The simple mastoidectomy - usually used as a canal wall up mastoidec- 
tomy-has some limitations. Extensive saucerization at the cortical portion can produce post-operative skin dimpling. Extensive removal of mastoid air cells may alter the homeostasis of gas exchange through the mastoid mucosa, resulting in tympanic membrane retraction [6]. If the patient has irreversible inflammatory mucosa in the mastoid air cells, removal of all air cells must be performed. However, if the mastoid mucosa does not contain pathologic tissues, or the mastoid air cells have been altered by new bone formation following sclerotic change, a simple mastoidectomy including complete removal of mastoid air cells is not the optimal surgical technique.

Conventionally, in trying to restore mastoid ventilation, the anterior epitympanic pathway through the supratubal recess and the posterior pathway through the incudo-stapedial space were ignored and replaced with a posterior tympanotomy. Posterior tympanotomy has proven to be an excellent approach to establish a ventilation pathway to the middle ear, but this approach risks facial nerve trauma and requires prolonged operative time [7]. To avoid the unnecessary removal of mastoid air cells and to gain the physiologic epitympanic pathway, we designed a new, minimally invasive approach to the epitympanum (Fig. 1, upper left).

Over the past 11 years, we have treated six patients who suffered traumatic facial nerve palsy (TFNP) involving only

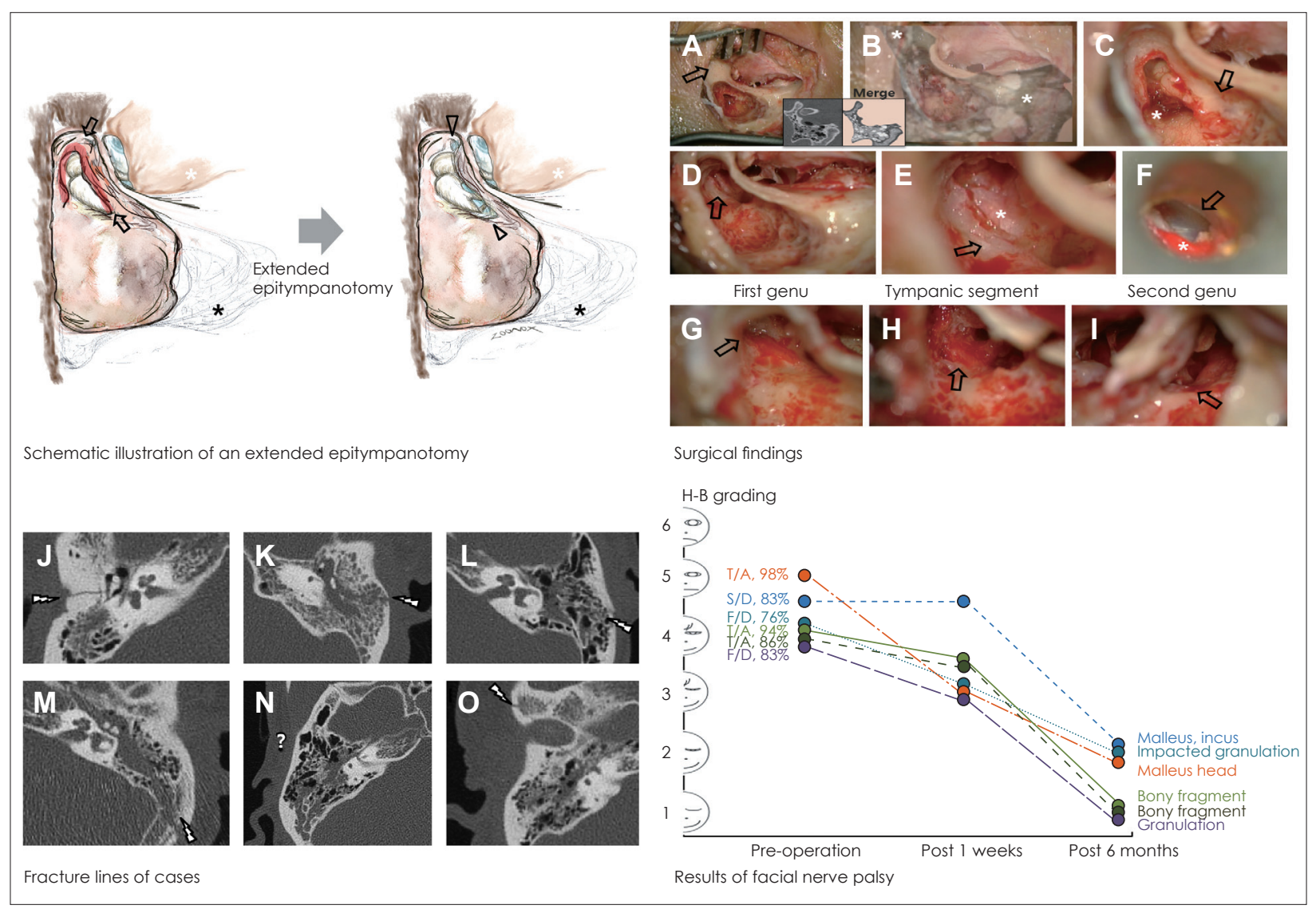

Fig. 1. Schematic illustration of an extended epitympanotomy. Upper left: A conventional epitympanotomy limits the exposure to the epior meso-tympanum. In an extended epitympanotomy, by drilling to the supratubal recess and the facial recess (black arrows), the surgical exposure is enhanced in the mesotympanum (black arrowheads). Canal incision was not performed (white asterisks) and mastoid air cell was removed minimally (black asterisks). Upper right: Surgical findings. (A) One arm of a self-retractor holding the incised temporalis muscle fibers out of the wound (black arrow). (B) About $30 \%$ of the mastoid air cells had been removed allowing the epitympanum to be approached, with preservation of the remaining air cells (white asterisks). (C) Cortical bone in contact with the epitympanum was drilled out using a diamond burr (black arrow). Pathologic tissue was found in the epitympanum (white asterisk). (D) The malleus head and incus were removed (black arrow). (E) Identification of the facial nerve (black arrow) evaluation of the pathologic condition (white asterisk). ( $F$ ) Intact tympanic membrane at completion of the surgical procedure (black arrow), and a single piece of Gelfoam and antibiotic ointment were placed inside the external auricular canal (white asterisk). (G-I) All portions of facial nerve from first genu and second genu were visible well (black arrow). Lower left: Fracture lines seen on temporal bone computed tomograms. (J) Definite fracture line that runs through the cortical bone of the lateral portion of the epitympanum (white thunder arrow). $(\mathrm{K}, \mathrm{L})$ Fracture line that runs through the mastoid air cells of the epitympanum (white thunder arrow). (M) Relatively wide fracture line at the epitympanum (white thunder arrow). (N) Partial haziness in mastoid air cells, but a definitive fracture line was not identified (white question mark). (O) Fracture line beginning at the anterior portion of the temporal bone (white thunder arrow). Lower right: Changing pattern of facial nerve palsy grading. All patients had recovered at post-operative 6 months. T/A: traffic accident, S/D: slip down, F/D: fall down, H-B: House-Brackmann. 
the tympanic segment. These cases were good candidates for the suggested techniques as mastoid pathologic changes did not exist, and the traumatic lesions were localized to the tympanic segment of the facial nerve. In each of these patients, we used this technique to minimize damage to the mastoid air-mucosa and facial canal. Based on our experience, we recommend this surgical approach for patients with TFNP in this anatomical location.

\section{Subjects and Methods}

\section{Participants}

A retrospective chart review of patients with TFNP, who underwent this technique between December 2007 and February 2017 at the Department of Otorhinolaryngology and Head and Neck Surgery, Chuncheon Sacred Heart Hospital, Hallym University, Korea was performed. A total of 6 patients were identified. In 3 patients, the source of trauma was a motor vehicle accident (one following collision, and two pedestrian vs. automobile). The source of trauma in the remaining three patients was secondary to falls. All patients were male, aged 13 to 83 years.

A database was created which included the degree of facial nerve paralysis [House Brackmann (H-B) grading system] at pre-operative, 1-week post-op, and 6-months post-op. All patients were followed with the periodic, post-operative H-B grading assessment. Only patients with TFNP localized to the tympanic segment with radiologic confirmation were included. Exclusion criteria were as follows: TFNP involving the labyrinthine or mastoid segment, facial nerve palsy secondary to COM or cholesteatoma, and patients with Bells palsy, Ramsey Hunt syndrome, or temporal bone fracture without facial nerve palsy. The Chuncheon Sacred Heart Hospital institutional review board approved the following study protocol (IRB number: 2018-3).

\section{Surgical technique}

The procedure was performed under general anesthesia. We placed the skin incision $1.5 \mathrm{~cm}$ posterior to the posterior auricular sulcus in the 8 to 11 o'clock direction. An anteriorly based periosteal flap (Palva flap) was elevated. To allow for subsequent epitympanic exposure, the incision along the temporalis line was extended anteriorly (Fig. 1A, arrow). Some temporalis muscle fibers located anteriorly were incised and cauterized using bi-polar cautery forceps. A self-retractor was positioned along the incised temporalis muscle fibers.

To minimize damage to the external auricular canal (EAC), a canal incision was not performed (white asterisks at Fig. 1 upper left). After elevation of the Palva flap, the periosteum was raised to the extent of the EAC skin elevation. In the EAC, the skin was elevated between the 2 and 7 o'clock direction. This permitted excellent surgical exposure at the superior quadrants of the mesotympanum, without tympanic membrane trauma. Elevation of the tympanomeatal flap was completed to the malleus umbo.

Next, a canal wall up mastoidectomy was performed. During this procedure, the initial drilling position was confirmed using a temporal bone computed tomogram. Drilling began just posterior to Henle's spine. The relatively large air cells under the mastoid cortical bone were exposed. The drilling epicenter was extended anteriorly for greater exposure, but not posteriorly (to the sigmoid sinus) or inferiorly (to the mastoid tip). After identification of the mastoid antrum and lateral semi-circular canal, removal of the mastoid air cells was begun in the epitympanic area (Fig. 1B).

The size of the air cells varies based on patients' pathology and the condition of the mastoid cavity. In present study, all patients had normal mastoid pneumatization, so exposing the area to the epitympanic lateral portion was relatively easy.

When the remaining cortical bone (Fig. 1C) that was in contact with the epitympanum was removed (Fig. 1D), the direction of the surgical microscope was repositioned to be parallel to the superior-posterior EAC. The exposed epitympanic cortical bone was drilled out with a small diamond burr. The drilling was extended to the facial recess portion. The superior-upper portion of the facial recess was drilled first (Fig. $1 \mathrm{C}$, arrow), so that prior identification of the facial nerve in the mastoid segment near the posterior tympanum was not necessary. Even though the posterior buttress was drilled out, the posterior incudal ligament was spared. As a result, stability of the ossicles was well maintained.

After checking the ossicles for pathological involvement, the incus and the heads of malleus were removed using a malleus nipper, to obtain the tissue-free space in the epitympanum for decompression (Fig. 1D, arrow). Next, Cog was removed from the supra-tubal recess. Bony fragments, pathologically positioned ossicles, and inflammatory granulation or fibrotic tissues around the exposed facial nerve, were removed (Fig. 1E). Through these maneuvers, the first to second genu of the facial nerve was decompressed (Fig. 1G-I), sparing the normal mastoid air cells, tympanic membrane, and a skin incision in the EAC. After facial nerve decompression, the elevated tympanomeatal flap was repositioned into its original position and antibiotic ointment was placed inside the EAC (Fig. 1F).

\section{Statistical analysis}

The Wilcoxon signed rank test was used to compare the de- 
gree of facial nerve paralysis pre- and post-surgery using the H-B grading system (a major statistical limitation as the H-B grading number is not a serial parameter). Statistical analyses were carried out using SPSS version 21 for Windows (IBM Corp., Armonk, NY, USA). $p<0.05$ was considered to be statistically significant for the analysis.

\section{Results}

\section{Patient and etiologic factors}

The age of the patients ranged from 13 to 83 years of age. The male:female ratio was 6:0, right:left ratio was 3:3. Two patients were injured from car crashes, and one was a pedestrian hit by an automobile. The other three patients slipped and fell (Table 1).

\section{Radiologic findings}

Otic capsule sparing fracture lines on Temporal bone computed tomogram were obtained in all patients (Fig. 1, lower left, J-O). Ossicular dislocations were identified in two patients (Fig. 1K and M). A definite fracture line was not visible in one patient (Fig. 1N). In one patient, a transverse fracture line was identified, using previously established criteria [8], that terminated at the epitympanic space (Fig. 1O).

\section{Surgical findings}

Various pathologic conditions of the tympanic area were identified. There was malleus and incus dislocation in one patient, with the dislocated malleus and incus impinging on the facial bony canal at the tympanic segment. Another patient had a malleus head dislocation, with surrounding granulation tissue. Bony fragments of the facial canal in the area of the tympanic segment of the facial nerve were checked and removed in 2 patients. Granulation tissue was observed in one patient between the facial canal and the ossicles (Fig. 1E, arrow). In one patient there was granulation tissue putting pressure on an intact facial canal, without a visible fracture line at the epitympanum (Table 1).

\section{Facial nerve recovery and hearing results}

Bilateral pre-operative differences in facial nerve electroneurography ranged from $76 \%$ to $98 \%$ (Table 1). The facial palsies of all patients were immediate types and the mean interval between the accident and the operation was 7.8 days (from 6 to 23 days). All enrolled patients had an H-B grade of 4 to 5 pre-operatively. Immediate changes in facial movements were checked in 5 patients $(p=0.041)$. Facial motions (incomplete closure of eye, to complete closure at effort) were measured in the anesthesia recovery room in 3 patients. In all cases, patients recovered facial nerve function with $\mathrm{H}-\mathrm{B}$ grading scores of 1 to 2 (long-term follow-up) $(p=0.024)$ (Fig. 1, lower right).

Staged ossiculoplasty was not performed for deafness (case 4) or for nearly deaf (case 1) patients. In one case (case 6), air-bone gaps did not exist in the follow-up period (air threshold: 57 decibels, bone 53). Staged ossiculoplasty was performed in one case (case 5, 14 months later, air threshold: 28, bone: 7), but hearing did not improve. One patient also refused ossiculoplasty (case 2, air: 46, bone: 10). Simultaneous ossiculoplasty with partial ossicular replacement prosthesis was performed in one case (case 3), and hearing returned to normal (air: 18, bone: 18).

\section{Discussion}

TFNP is relatively rare in cases of head trauma. Previously, TFNP was categorized by the direction of the fracture line (longitudinal and transverse fractures). Recently this classification

Table 1. Patients' demographics

\begin{tabular}{|c|c|c|c|c|c|c|c|c|c|c|}
\hline & $\begin{array}{c}\text { Age } \\
\text { (years) }\end{array}$ & Sex & Site & Cause & $\begin{array}{c}\text { ENoG } \\
(\%)\end{array}$ & $\begin{array}{l}\text { Immediate } \\
\text { or delayed } \\
\text { palsy }\end{array}$ & Injury site & $\begin{array}{l}\text { Accident- } \\
\text { operation } \\
\text { interval } \\
\text { (days) }\end{array}$ & $\begin{array}{l}\text { Operative } \\
\text { finding }\end{array}$ & $\begin{array}{c}\text { Pre-operative } \\
\mathrm{H}-\mathrm{B} \text { grading }\end{array}$ \\
\hline Case 1 & 83 & Male & Right & Slip down & 83 & Immediate & Tympanic segment & 6 & $\begin{array}{l}\text { Dislocated malleus } \\
\text { and incus }\end{array}$ & 4 or 5 \\
\hline Case 2 & 20 & Male & Left & Fall down & 83 & Immediate & lst genu & 18 & Granulation tissue & 4 \\
\hline Case 3 & 13 & Male & Left & Out-car & 94 & Immediate & Tympanic segment & 23 & $\begin{array}{l}\text { Bony fragment of } \\
\text { facial canal }\end{array}$ & 4 \\
\hline Case 4 & 39 & Male & Left & Out-car & 98 & Immediate & Tympanic segment & 12 & Dislocated malleus & 5 \\
\hline Case 5 & 39 & Male & Right & In-car & 86 & Immediate & Tympanic segment & 9 & $\begin{array}{l}\text { Bony fragment of } \\
\text { facial canal }\end{array}$ & 4 \\
\hline Case 6 & 61 & Male & Right & Fall down & 76 & Immediate & No identification & 10 & Granulation tissue & 4 \\
\hline
\end{tabular}

ENoG: electroneuronography, H-B: House-Brackmann 
was modified to include otic capsule sparing vs. involvement [9]. With either classification, functional loss in TFNP is important to both clinicians and patients. Our 6 cases were unusual, since all involved the facial tympanic segment and spared the otic capsule.

Longitudinal fractures were seen in 5 cases, and a transverse fracture line was observed in 1 case. The degree of facial nerve paralysis was severe, but the damage to the facial nerve seen intra-operatively was relatively mild.

Conventionally, wide exposure of the facial nerve from the lateral portion of the labyrinthine to the mastoid segment has been recommended, with an $86 \%$ success rate for $\mathrm{H}-\mathrm{B}$ grades 1-3 [1,3]. An epineurium incision has been recommended by some clinicians [1,5]; however, in the present series of cases, we determined the exact location of damage to the facial nerve and only decompressed the involved area. Bony fragments or dislocated ossicles were the main problems we encountered and by meticulously removing these displaced structures we could expose the facial nerve and restore its normal contour. Removing the malleus head and incus created a large space in the epitympanum, and the enlarged epitympanic space permitted sufficient space for healing during the recovery period.

For this approach to facial nerve decompression, we applied the techniques used in a conventional mastoidectomy to remove most mastoid air cells. However, the extended air cells near the zygomatic arch were ignored.

The alternative procedures have major limitations. Opening of the facial recess can require a prolonged amount of time and risks facial nerve injury. In addition, if the pathology is localized to the epitympanum, otologic surgeons may need to modify the conventional approach for exposure. In the present study, we demonstrate that an extended epitympanotomy with minimal mastoid air cell removal (black asterisks at Fig. 1 upper left) can be used to treat patients with TFNP.

Other options have been recommended in the literature. A transcanal atticotomy via a transcanal approach was used to resect epitympanic pathologic tissue [10]. However, that technique has limitations when approaching the tegmen tympani, when using straight instruments. To mitigate these limitations, some clinicians have modified the approach using endoscopy via a transcanal approach [11]. Although several different approaches have been tried and developed, the transmastoid approach remains the best way to ensure a clear view. As mastoid aeration was normal in these six cases of TFNP, we drilled to the temporal line allowing a direct view to the epitympanum.

Negative pressure in the mastoid can induce retraction pocket formation [12]. However, there has been debate on whether mastoid aeration after mastoidectomy is helpful or harmful [6]. Some authors has suggested that negative pressure from the large air space will induce tympanic membrane retraction. In contrast, other authors have suggested that limited aeration of the mastoidectomy site may lead to retraction [13]. We applied this same surgical approach (an extended epitympanotomy) to pre-cholesteatoma patients and also applied an extended epitympanotomy with complete removal of mastoid air cells to other COM patients. There were many advantages of this operative modification compared to a conventional mastoidectomy (Fig. 2). First, opening of the facial recess was not necessary. Theoretically, opening of the facial recess is a bypass gate for air ventilation. In the present procedure, the nor-

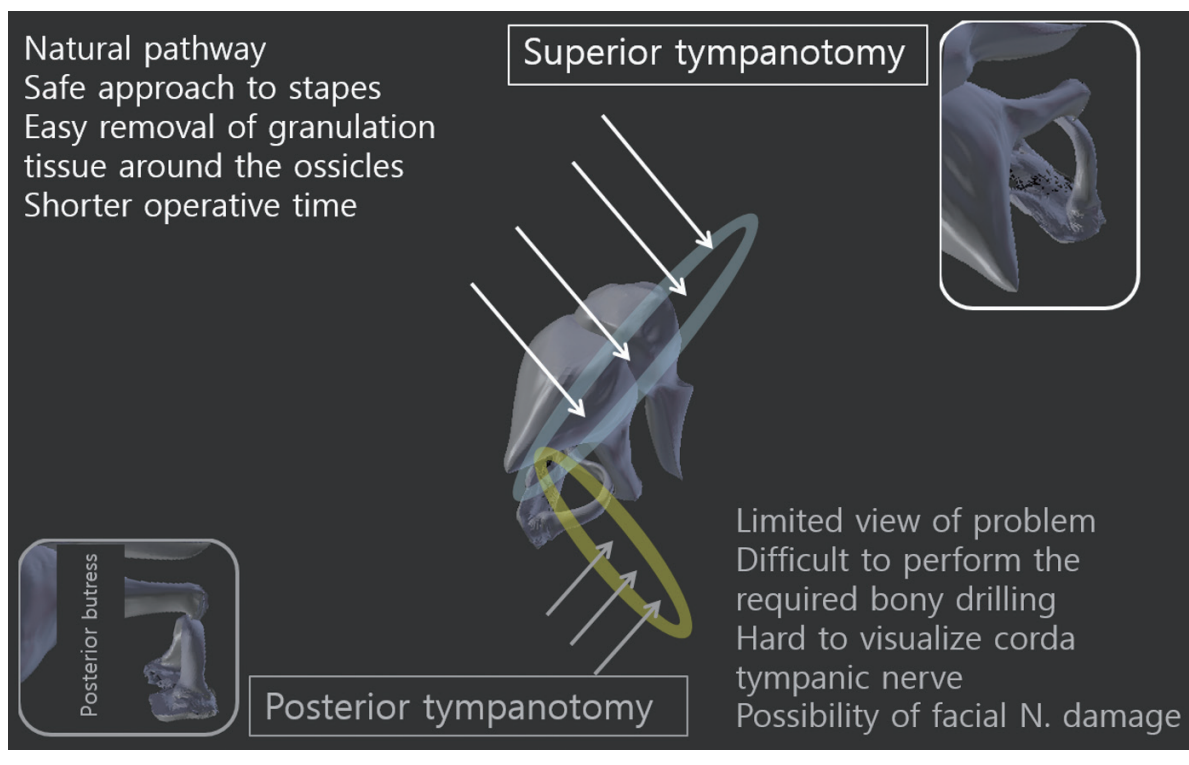

Natural pathway

Safe approach to stapes

Easy removal of granulation

tissue around the ossicles

Shorter operative time
Fig. 2. Advantages of extended epitympanotomy (superior tympanotomy), compared with posterior tympanotomy. N: nerve. 
mal pathways of the temporal bone (anterior and posterior epitympanic pathway) were maintained or re-opened by removal of granular tissue around the ossicles and Cog (black arrowheads at Fig. 1 upper left, compared with black arrows at Fig.1 upper left as conventional mastoidectomy) [14]. Additionally, Prussak's space was widen by drilling the cortical bone between the ossicles and the superior EAC. Extended mastoid drilling to the temporalis line allowed us to visualize the supra-tubal recess without removing the malleus head. Finally, overall surgical time was shorter because the extra and unnecessary steps could be avoided.

In this study, we performed our technique on six patients with TFNP. Unfortunately, given the small number of cases in this series (6 cases), it is difficult to compare this approach to other surgical methods relative to facial nerve recovery. When we compared with other studies on the conventional transmastoid approach, the following was seen: $86 \%$ success in failed patients with the conservative treatment [3], 100\% success rate with adding of the temporal lobe retraction [1], and $83.3 \%$ success rate in patients with perigeniculate ganglion injury [15]. The recovery rate for our technique was $100 \%$ (pre-operative H-B grade: 4 to 5, post-operative H-B grade: 1 to 2). Even though the success rates are relatively similar, extended epitympanotomy has the advantages of greater simplicity and shorter operation time. We recommend it for patients with facial nerve paralysis located in the epitympanum.

In conclusion, extended epitympanotomy is very useful to achieve an ideal airflow pathway and optimal surgical view to the epitympanum. Based on our experience, we believe that it is the proper method to treat facial nerve palsy due to trauma.

\section{Acknowledgments}

Illustrations in Fig. 1 and 2 are by Dr. Jun Ho Lee (Corresponding Author, Department of Otorhinolaryngology and Head and Neck Surgery, Chuncheon Sacred Heart Hospital, College of Medicine, Hallym University, Korea). This work was supported by the National Research Foundation of Korea (NRF) grant funded by the Korea government (NRF-2016R1D1A1B01014128), Republic of Korea.

\section{Conflicts of interest}

The authors have no financial conflicts of interest.

\section{Author Contributions}

Conceptualization: Jun Ho Lee. Data curation: Jun Ho Lee. Formal analysis: Jun Ho Lee. Funding acquisition: Jun Ho Lee. Investigation: Jun Ho Lee. Methodology: Jun Ho Lee. Project administration: Jun Ho Lee. Resources: Jun Ho Lee. Software: Jun Ho Lee. Supervision:
Jiwon Chang. Validation: Jiwon Chang. Visualization: Jun Ho Lee. Writing — original drift: Janet Ren Chao. Writing — review \& editing: Janet Ren Chao and Jun Ho Lee.

\section{ORCID iDs}

Janet Ren Chao

Jiwon Chang

Jun Ho Lee

https://orcid.org/0000-0002-8209-0892

https://orcid.org/0000-0003-1660-1831

https://orcid.org/0000-0002-8668-7047

\section{REFERENCES}

1) Kim MW, Ryu NG, Lim BW, Kim J. Temporal lobe retraction provides better surgical exposure of the peri-geniculate ganglion for facial nerve decompression via transmastoid approach. Yonsei Med J 2016;57:1482-7.

2) Cannon RB, Thomson RS, Shelton C, Gurgel RK. Long-term outcomes after middle fossa approach for traumatic facial nerve paralysis. Otol Neurotol 2016;37:799-804.

3) Yadav S, Panda NK, Verma R, Bakshi J, Modi M. Surgery for posttraumatic facial paralysis: are we overdoing it? Eur Arch Otorhinolaryngol 2018;275:2695-703.

4) Kahinga AA, Han JH, Moon IS. Total transcanal endoscopic facial nerve decompression for traumatic facial nerve palsy. Yonsei Med J 2018;59:457-60.

5) Sun DQ, Andresen NS, Gantz BJ. Surgical management of acute facial palsy. Otolaryngol Clin North Am 2018;51:1077-92.

6) Lee HJ, Chao JR, Yeon YK, Kumar V, Park CH, Kim HJ, et al. Canal reconstruction and mastoid obliteration using floating cartilages and musculoperiosteal flaps. Laryngoscope 2017;127:1153-60.

7) Ryu NG, Kim J. How to avoid facial nerve injury in mastoidectomy? J Audiol Otol 2016;20:68-72.

8) Yanagihara N, Murakami S, Nishihara S. Temporal bone fractures inducing facial nerve paralysis: a new classification and its clinical significance. Ear Nose Throat J 1997;76:79-80, 83-6.

9) Dahiya R, Keller JD, Litofsky NS, Bankey PE, Bonassar LJ, Megerian CA. Temporal bone fractures: otic capsule sparing versus otic capsule violating clinical and radiographic considerations. J Trauma 1999;47:1079-83.

10) Bernardeschi D, Russo FY, Nguyen Y, Canu G, Mosnier I, De Seta D, et al. Management of epi- and mesotympanic cholesteatomas by one-stage trans-canal atticotomy in adults. Eur Arch Otorhinolaryngol 2016;273:2941-6.

11) Alicandri-Ciufelli M, Marchioni D, Kakehata S, Presutti L, Villari D. Endoscopic management of attic cholesteatoma: long-term results. Otolaryngol Clin North Am 2016;49:1265-70.

12) Palva T, Virtanen H. Ear surgery and mastoid air cell system. Arch Otolaryngol 1981;107:71-3.

13) Seong CS, Cho TH, Tark GS, Lee UY. A study on the Eustachian tube function and mastoid hypocellurality in chronic otitis media. Korean J Otolaryngol 1983;26:199-207.

14) Palva T, Ramsay H, Böhling T. Tensor fold and anterior epitympanum. Am J Otol 1997;18:307-16.

15) Liu Y, Liu S, Li J, Chen X, Sun J, Li Y. Management of facial palsy after temporal bone fracture via the transmastoid approach. Acta Otolaryngol 2015;135:307-11. 